\section{Factors Associated With Late HIV Diagnosis among Peoples Living with HIV, Northwest Ethiopia: Hospital based Unmatched Case-control Study}

\section{Abstract}

Background: Early HIV diagnosis and access to treatment is one of the most effective ways to prevent its further spread and to protect the health of those living with the virus. However, delay in diagnosis is the major risk factor for uptake of and response to antiretroviral therapy.

Methods: Institution-based unmatched case-control study design was used in the study. The study was conducted in Debre-Markos and Finote-Selam Hospitals, Northwest Ethiopia. Cases were people living with HIV who had CD4 count $<350$ cells $/ \mathrm{mm}^{3}$ or WHO clinical stage III and IV regardless of the CD4 count at first presentation and controls were those who had CD4 count $\geq 350$ cells $/ \mathrm{mm}^{3}$ or WHO clinical stage I and II. If both criteria were available, the CD4 count was preferred as recommended by World Health Organization. A total of 392 respondents [196 cases and 196 controls] were recruited and selected systematically. The data were collected by trained nurses using chart review and interviewer administered structured questionnaire. Binary Logistic Regression Model was used to identify the factors associated with late HIV diagnosis.

Findings: out of the total of 392 participants, 376 [187 cases and 189 controls] people living with HIV provided complete response. Having no understanding, compared to having understanding, about HIV/AIDS [AOR=1.7, 95\% $\mathrm{Cl}=1.08-2.79$ ] and $A R T$ [AOR=2.1, 95\%Cl: 1.25-3.72], being tested as a result of symptoms/illness, compared to being tested for risk exposure [inverted $\mathrm{AOR}=2.5,95 \% \mathrm{Cl}: 1.64-4.76$ ], and acquiring HIV through sexual contact, compared to acquiring it through other modes $[A O R=2.5,95 \% \mathrm{Cl}=1.52-4.76]$ were positively and independently associated with late HIV diagnosis.

Conclusions: Unlike perceived HIV stigma, having no understanding about HIV and ART, being tested for presence of symptoms/illness, and acquiring HIV through sexual contact were independent and significant factors for late HIV diagnosis.

Keywords: Late HIV diagnosis; Associated factors; Unmatched case-control; Ethiopia

Abbreviations: AIDS: Acquired Immuno-Deficiency Syndrome; ART: Anti-Retroviral Treatment; CDC: Center for Disease Control and Prevention; EDHS: Ethiopian Demographic and Health Survey; HCT: HIV Counseling and Testing; HIV: Human Immunodeficiency Virus; PIHCT: Provider Initiated HIV Counseling and Testing; PITC: Provider Initiated Testing and Counseling; SPSS: Software Package for Social Sciences; STIs: Sexually Transmitted Infections; VCT: Voluntary Counseling and Testing; WHO: World Health Organization
Abebayehu Bitew Aniley', Tadesse Awoke Ayele ${ }^{2}$, Ejigu Gebeye Zeleke ${ }^{2}$ and Assefa Andargie Kassa ${ }^{1}$

1 Department of Public Health, College of Medicine and Health Sciences, Wollo University, Ethiopia

2 Department of Epidemiology and Biostatistics, Institute of Public Health, College of Medicine and Health Sciences, University of Gondar, Ethiopia

\section{Corresponding author:}

Abebayehu Bitew Aniley

\section{झ abebayehubitew@gmail.com}

Department of Public Health, College of Medicine and Health Sciences, Wollo University, Dessiee, P.O. Box: 1145, Ethiopia

Tel: +251-92-1528949

Citation: Aniley AB, Ayele TA, Zeleke EG, et al. Factors Associated With Late HIV Diagnosis among Peoples Living with HIV, Northwest Ethiopia: Hospital based Unmatched Casecontrol Study. J HIV Retrovirus. 2016, 2:1. 


\section{Introduction}

For over twenty years, HIV infection and AIDS have been significant public health problems particularly in low- and middle- income countries, with two-thirds of the world's HIV-infected population living in Africa [1]. It is also one of the most serious public health and development challenges in Ethiopia [2].

Early diagnosis and access to treatment is one of the most effective ways to prevent the further spread of HIV and to protect the health of those living with the virus [3]. It helps people with HIV to timely get and appropriately use antiretroviral treatment. The timely and appropriate use of antiretroviral treatment lowers the amount of virus in their body and can dramatically reduce their risk of morbidity, mortality and it has been conclusively shown to decrease HIV transmission by greater than $90 \%[4,5]$.

There is quicker and easier HIV testing, and more people have been tested and know more about how to prevent HIV infection today than at any other time in the history of the disease. However, the presence of too few people with HIV who are aware of their infection remains being one of the significant challenges of infection prevention strategies. Because, the majorities of people with HIV who do not know they are infected are significantly transmitting HIV and contribute half of the new infections [3]. Delay in knowing the HIV positive status also results the advancement of the disease which poses a less favorable clinical course, with reduced or incomplete treatment response, more rapid clinical progression, and higher risk of mortality on the victims. In addition, it generates a considerable and avoidable resource burden to the healthcare system [5].

Many factors are found to keep people from finding out their HIV status and determine the future course of its epidemic. These include socio-demographic factors like older age, male gender, living in rural areas and behavioral factors like HIV/AIDS and ART-related knowledge, attitude and belief, social stigma, risk behavior modification, access to high-quality services for sexually transmitted infections [STIs], provision and uptake of HIV counseling and testing, and access to antiretroviral therapy [ART] [5-20].

\section{Methods}

Unmatched case-control study was conducted from April 15 to March 20, 2013 in Debre-Markos and Finote-Selam Hospitals, Northwest Ethiopia. Debre-Markos and Finote-Selam are the capitals of East Gojjam and West Gojjam Zones in Amhara Regional state, respectively. Debre-Markos is found $300 \mathrm{~km}$ northwest of the capital of the Country, Addis Ababa and 265 $\mathrm{km}$ Southeast of Bahir Dar, the capital of Amhara Regional state. Similarly, Finote-Selam is located $387 \mathrm{~km}$ northwest of Addis Ababa and $166 \mathrm{~km}$ southeast of Bahir Dar. The two hospitals provide Voluntary Counseling and Testing [VCT], Prevention of Mother to Child Transmission [PMTCT], ART and treatment of opportunistic infection services. The study population were cases and controls of people with HIV, age $\geq 18$ years, who visited the ART clinics of the two hospitals during the data collection period. Cases were individuals who had CD4 cell count $<350$ cells $/ \mathrm{mm}^{3}$ or those who had WHO clinical stage III or IV at their presentation during first HIV diagnosis. Controls were individuals who had CD4 cell count $\geq 350$ cells $/ \mathrm{mm}^{3}$ or those who had WHO clinical stage I or II at their presentation during first HIV diagnosis. Where clinical and immunological classifications were both available, immune status, reflected by CD4 count, was preferred as recommended by WHO [21-23].

The sample size was calculated using Epi info version 7 developed by Communicable Disease Control after considering the following assumptions: proportion of high perceived HIV stigma were $9.4 \%$ and $22.5 \%$ among controls and cases respectively, $95 \% \mathrm{Cl}, 90 \%$ power, 1 to 1 case to control ratio and $10 \%$ non-response rate. The total sample size was 392 [196 cases and 196 controls]. Perceived HIV stigma was among the independent factors associated with late HIV diagnosis, which provided large sample size. A study conducted in South Wollo, Northeast Ethiopia, showed that respondents who had high perceived stigma, as compared to those who had low perceived stigma, were more likely to be diagnosed late at first presentation [14]. Cases and controls were recruited using systematic random sampling technique after the total sample size was allocated proportionally to the Hospitals based on the ART clients load. Therefore, 262 respondents [131 cases and 131 controls] were recruited from Debre-Markos Hospital and the remaining 130 respondents [65 cases and 65 controls] were selected from Finote-Selam Hospital. Unique patients' identification number in the ART clinics was used as a sampling frame.

The data was collected by trained nurses working in the Hospitals after informed written consent from each respondent was obtained. The data collection tool was pre-coded, pretested and interview-based structured questionnaire developed after reviewing relevant literatures on the issue. It consisted of socio-demographic factors, comprehensive HIV knowledge, understanding about HIV/AIDS, HIV risk perception and risky behaviors, perceived HIV stigma, knowledge and understanding about ART, medical history and factors related to healthcare service and HIV testing. Study participants provided the response to all questions based on the information they had before or during their diagnosis at first presentation.

Comprehensive HIV knowledge was determined based on 5 items which were used in Ethiopian Demographic and Health Survey [EDHS] 2011, which is part of the worldwide MEASURE Demographic and Health Survey project funded by the United States Agency for International Development [USAID] and implemented by the Ethiopian Central Statistical Agency. An individual who correctly answered all of the items was considered as having comprehensive HIV knowledge, but if $s /$ he failed to answer at least 1 of them, s/he was considered as $\mathrm{s} /$ he didn' $\mathrm{t}^{\prime}$ have it [2]. Similarly, attitude of respondents, towards people with HIV/AIDS was measured using 4 "yes/no" attitude questions used by EDHS. One point was given for "yes" responses and zero point for "no" responses. The total score of a respondent, therefore, ranged from 0 to 4 . Scores of individuals less than the mean score of the study population was categorized as having "negative" attitude and scores greater than or equal to the mean score represented "positive" attitude [2]. Understanding about HIV/AIDS was also determined by 5 "yes/no" HIV/AIDS questions 
adopted from related literatures. "Yes" responses, given 1 point, indicated rejecting the common truth about HIV/AIDS, while "no" response, given 0 point, indicated accepting it. Based on a similar technique used in the determination of attitude about HIV/AIDS, it was categorized as "good" or "poor". In addition, perceived HIV stigma, categorized as "high" or "low", was measured using 14 "yes/no" items which were validated and recommended in different studies. These items were related to participants' perceptions, about how their partners, friends, families and the community would react to them if their HIV positive status was known [24-26]. Further, knowledge about ART was measured using 6 "yes/no" items and labeled as "good" or "poor" based on the total score. The level of understanding about ART was also determined using 6 "yes/no" items and categorized as "have no understanding" and "have understanding" using similar techniques.

To maintain the quality of data obtained, the questionnaire was translated to "Amharic", the local language, and back to English language to check its consistency by individuals who had related experience. In addition, the questionnaire was pretested and there was regular supervision and timely check-up of the completeness and consistency of responses to questions throughout the data collection period. Ethical approval was obtained from the Ethical Review Board of University of Gondar and permission letter was also received from the Hospital Administrators.

The data were entered into a pre-designed format in Epi Ifo version 3.5.1 developed by Communicable Disease Control and transferred to SPSS version 16 for analysis. Binary logistic regression analysis was applied to identify the independent and significant factors. Before running the regression analysis, assumptions to apply binary logisic regression were checked and satisfied. These include ratio of cases to independent variables [greater than 10 to 1], overall relationship of independent variables with the dependent variable [Model chi-square, $p$-value $<0.05]$, Hosmer and Lemshow test of model goodness of fit [p-value $>0.05$ ] and the standard errors of model coefficients were less than 2 .

Description of the main analysis findings was done using frequencies, percentages and summary statistics. To identify the factors associated with late HIV diagnosis, first, uni-variate analysis, in the binary logistic regression, between each independent variable and the dependant one was carried out. Those variables with a $p$-value $\leq 0.2$ were then included in the multiple analysis of binary logistic regression to decrease the effect of residual confounding. In the multiple analysis, the Backward Likelihood Ratio Method was used to identify the independent and significant factors at 0.05 level of significance. Odds ratio and $95 \%$ confidence interval were used in the interpretation of the result.

\section{Results}

\section{Socio-demographic characteristics}

Out of the total of 392 people living with HIV [196 cases and 196 controls], 376 [96\% response rate] provided appropriate data. Out of these, 135 [35.9\%] were males and 241 [64.1\%] were females and 187 [49.7\%] were cases and 189 [50.3\%] were controls. Among the cases, 123 [65.8\%] were from Debre-Markos and 64 [34.2\%] were from Finote-Selam Hospitals. Similarly, among the controls, 125 [66.1\%] and 64 [33.9\%] were from Debre-Markos and Finote-Selam Hospitals respectively.

\section{Factors associated with late HIV diagnosis}

Factors which were associated with late HIV diagnosis in the univariate logistic regression at a 0.2 level of significant include age, presence of comprehensive HIV knowledge, attitude towards people living with HIV/AIDS, understanding about HIV/AIDS, perceived HIV stigma, knowledge about ART, understanding about ART, number of sexual partners before HIV positive diagnosis, main reason for wanting to test, mode of HIV acquisition, time of HIV negative test result before the positive diagnosis, receiving treatment from medical clinic, receiving treatment from pharmacy/drug store, presence of hospital admission, presence of opportunistic illness, time of latest HIV negative test result, year of HIV positive diagnosis, and distance of the nearest healthcare facility. However, religion and marital status were not significantly associated with late HIV diagnosis.

These factors were included in the multiple logistic regression analysis. Variables which were significantly associated with Late HIV Diagnosis at a 0.05 level of significance in the multiple logistic regression analysis were belief about HIV/AIDS, belief about ART, main reason for wanting to test, mode of HIV acquisition and year of HIV positive diagnosis. However, the primary hypothesized factor, perceived HIV stigma, was marginally significant.

Respondents who had no understanding about HIV/AIDS were about 1.7 times more likely to be diagnosed late as compared to those who had understanding [AOR=1.7, 95\% $\mathrm{Cl}=1.08-2.79]$. Similarly, individuals who had no understanding about ART were about 2 times at risk of late HIV diagnosis as compared to those who had understanding $[\mathrm{AOR}=2.1,95 \% \mathrm{Cl}=1.25-3.72]$.

In addition, study participants who were tested as a result of HIV risk exposure had $60 \%$ decreased risk of being diagnosed late as compared to those tested due to presence of symptoms/illness [AOR=0.4, 95\% $\mathrm{Cl}: 0.21-0.61]$. Even though it was marginally significant, participants tested for screening purpose, as compared to those tested due to presence of symptoms/illness, had $50 \%$ decreased risk of being diagnosed late $[A O R=0.5,95 \% \mathrm{Cl}$ : 0.27-1.06].

As compared to those who acquired HIV through sexual contact, respondents who acquired HIV through other modes of transmission including sharp materials, blood transfusion, intravenous drug use and wound contact had $50 \%$ decreased risk of being diagnosed late $[\mathrm{AOR}=0.4,95 \% \mathrm{Cl}=0.21-0.66]$.

Study participants who were tested after 2008 had $70 \%$ decreased risk of late HIV diagnosis as compared to individuals who were tested before 2007 [ $A O R=0.3,95 \% \mathrm{Cl}=0.17-0.72$ ]. However, there was no significant difference between those diagnosed before 2007 and 2007-2008 [AOR=0.6, 95\% Cl=0.3-1.42]. 


\section{Discussion}

Diagnosing HIV at its earlier stage has easily recognizable importance for those living with it to timely link with and gets maximal benefit from HIV care and treatment service. It also helps to strengthen actions taken to prevent transmission of the infection particularly from those who are unaware of their infection. However, different factors are identified to hinder early HIV diagnosis.

The primary hypothesized factor for late HIV diagnosis in this study was perceived HIV stigma. It was marginally significant in the multiple logistic regression analysis result. Respondents who had high perceived HIV stigma, as compared to those who had low perceived HIV stigma, were 1.7 times more likely to be diagnosed [AOR=1.7, 95\% $\mathrm{Cl}: 1-2.89]$, which is similar with the result of a case-control study in South Wollo, Northeast Ethiopia [14]. Findings evidenced that there was consistent improvement in the immune status of people living with HIV at their first diagnosis across time trends because of increased knowledge about HIV/AIDS and access to HIV testing and counseling services $[9,27]$.

In addition, the earlier study considered individuals who were greater or equal to 15 years old; while this study included individuals greater or equal to 18 years old. Individuals with age 15-17 years old are usually considered as they are immature by the society and they might feel that their parents, families and the society would stigmatize them due to their HIV acquisition in their earlier age. This, as a result, might increase perceived HIV stigma.

Study participants who had no understanding about HIV/AIDS were more likely to be diagnosed late at first presentation as compared to those who had understanding. This could be due to lack of comprehensive HIV knowledge, which was significant predictor of late HIV diagnosis in the univariate analysis, even if it was not in the multivariate one. Because, individuals who had no understanding about HIV/AIDS, compared to those having understanding, had no comprehensive HIV knowledge [COR=2.6, $95 \% \mathrm{Cl}=1.57-4.27]$. A cases-case comparison study conducted in Venezuela supported this possible explanation which identified low knowledge of HIV/AIDS being the main barriers to HIV testing [13].

Similarly, participants who had poor belief about ART were more likely to be diagnosed late as compared to those who had good ART belief, which is strengthened by a case-control study finding in South Wollo that identified believing ART has side effects increased late presentation to HIV care.

In addition, individuals who were tested due to the presence of symptoms or illness were more likely to be diagnosed late for HIV as compared to those tested for both HIV risk exposure and screening purpose. This could be due to the difference in the time interval between infection and diagnosis. Because, presence of symptoms or illness usually come in the later stage of HIV infection. This result is consistent with a finding in New Zealand [18] and South Korea [11].

Sexual contact, as compared to other modes of HIV transmission, was also found to be another risk factor for late HIV diagnosis at first presentation. The possible justification for this could be the high proportion of people living with HIV who acquired it through sexual contact, perceiving that they had HIV as a result of their immoral act and fear of reactions from other people.

On the other hand, those who acquired HIV via other modes of HIV transmission might not believe that they contracted due to their sin. This finding is similar with study results from India and Brussels and France $[7,10]$.

\section{Strengths and limitations of the study}

Despite many studies on HIV related problems, there was no study conducted on factors associated with late HIV diagnosis in Ethiopia before. Therefore, this study would probably be the first on this specific issue. It also tried to identify possible factors through reviewing related literatures.

Since this study was based on a case-control design, there would be recall bias that might affect the actual result. All the study participants were also selected without specification of their year of first HIV positive diagnosis which could increase the recall bias. However, to optimize this problem, the interviewers were trained on the nature of the questions and respondents were also given time to remember the information during the interview.

\section{Conclusion}

The primary hypothesized factor, perceived HIV stigma, was not independent and significant predictor of late HIV diagnosis. However, having poor belief about HIV/AIDS and ART, wanting to test as a result of presence of symptoms or illness, and acquiring HIV through sexual contact were independent and significant factors associated with late HIV diagnosis among people living with HIV in Debre-Markos and Finote-Selam hospitals (Table 1).

\section{Author's Contributions}

ABA participated in the design of the study and the questionnaire, coordinated the data collection process, performed the statistical analysis, interpreted the data and wrote the first draft of the manuscript. TAA, EGZ and AAK had revised it critically for important intellectual content. All authors edited and approved the final version of the manuscript.

\section{Acknowledgements}

We would like to thank University of Gondar, Institute of Public Health for giving this opportunity and USAID ENHAT-CS program, which is funded by PEPFAR and implemented by an MSH led consortium of international and Ethiopian organizations for its financial support.

Our heartfelt thank is also extended to Debre-Markos and FinoteSelam hospital administrators, data collectors, supervisors and respondents for their kind cooperation.

\section{Disclaimer}

This publication was made possible by the generous support of the United States Agency for International Development [USAID] under RFA: 663-11-000005. The contents are the responsibility of the authors and do not necessarily reflect the views of USAID or the United States Government. 


\section{References}

1 Bendavid E, Young SD, Katzenstein DA, Bayoumi AM, Sanders GD, et al. (2008) Cost-effectiveness of HIV monitoring strategies in resource-limited settings: a southern African analysis. Arch Intern Med 168: 1910-1918.

2 http://www.dhsprogram.com/

3 http://www.cdc.gov

4 Cohen MS, Chen YQ, McCauley M, Gamble T, Hosseinipour MC, et al. (2011) Prevention of HIV-1 infection with early antiretroviral therapy. N Engl J Med 365: 493-505.

5 D'Arminio Monforte A, Antinori A, Girardi E, Ceccherini-Silberstein F, Marchetti G, et al. (2012) HIV-Infected Late Presenter Patients. AIDS Res Treat 2012: 902679.

6 Schwarcz S, Hsu L, Dilley JW, Loeb L, Nelson K, et al. (2006) Late diagnosis of HIV infection: trends, prevalence, and characteristics of persons whose HIV diagnosis occurred within 12 months of developing AIDS. J Acquir Immune Defic Syndr 43: 491-494.

7 Ndiaye B, Salleron J, Vincent A, Bataille P, Bonnevie F, et al. (2011) Factors associated with presentation to care with advanced HIV disease in Brussels and Northern France: 1997-2007. BMC Infect Dis 11: 11 .

8 Narcisse E, Marie-Therese GS, Mathieu N (2012) Risk Factors for Late Presentation for Care among HIV-Infected Patients in Guadeloupe: 1988-2009. J AIDS Clinic Res 3: 166.

9 Metallidis S, Pilalas D, Skoura L, Haidich AB, Tsachouridou O, et al. (2012) Time trends and correlates of late presentation for HIV care in Northern Greece during the decade 2000 to 2010. J Int AIDS Soc 15: 17395.

10 Mojumdar K, Vajpayee M, Chauhan NK, Mendiratta S (2010) Late presenters to HIV care and treatment, identification of associated risk factors in HIV-1 infected Indian population. BMC Public Health 10: 416 .

11 Lee JH, Kim GJ, Choi BS, Hong KJ, Heo MK, et al. (2010) Increasing late diagnosis in HIV infection in South Korea: 2000-2007. BMC Public Health 10: 411.

12 Thanawuth N, Chongsuvivatwong V (2008) Late HIV diagnosis and delay in CD4 count measurement among HIV-infected patients in Southern Thailand: Psychological and Socio-medical Aspects of AIDS/HIV. AIDS Care 20: 43-50.

13 Bonjour MA, Montagne M, Zambrano M, Molina G, Lippuner C, et al. (2008) Determinants of late disease-stage presentation at diagnosis of HIV infection in Venezuela: a case-case comparison. AIDS Res Ther 5: 6 .
14 Abaynew Y, Deribew A, Deribe K (2011) Factors associated with late presentation to HIV/AIDS care in South Wollo ZoneEthiopia: a casecontrol study. AIDS Res Ther 8: 8.

15 Kigozi Isaac M, Dobkin Loren M, Martin Jeffrey N, Geng Elvin H, Muyindike W, et al. (2009) Late disease stage at presentation to an HIV clinic in the era of free antiretroviral therapy in sub-Saharan Africa. J Acquir Immune Defic Syndr 52: 280-289.

16 Medley A, Ackers M, Amolloh M, Owuor P, Muttai H, et al. (2013) Early uptake of HIV clinical care after testing HIV-positive during home-based testing and counseling in western Kenya. AIDS Behav 17: 224-234.

17 Hatcher AM, Turan JM, Leslie HH, Kanya LW, Kwena Z, et al. (2012) Predictors of linkage to care following community-based HIV counseling and testing in rural Kenya. AIDS Behav 16: 1295-1307.

18 Dickson N, McAllister S, Sharples K, Paul C (2012) Late presentation of HIV infection among adults in New Zealand: 2005-2010. HIV Med 13: $182-189$.

19 Wanyenze RK, Kamya MR, Fatch R, Mayanja-Kizza H, Baveewo S, et al. (2011) Missed opportunities for HIV testing and late-stage diagnosis among HIV-infected patients in Uganda. PLoS One 6: e21794.

20 Yang B, Chan SK, Mohammad N, Meyer JA, Risser J, et al. (2010) Late HIV diagnosis in Houston/Harris County, Texas, 2000-2007. AIDS Care 22: 766-774.

21 Antinori A, Coenen T, Costagiola D, Dedes N, Ellefson M, et al. (2011) Late presentation of HIV infection: a consensus definition. HIV Med 12: 61-64.

22 UK Collaborative HIV Cohort (UK CHIC) Steering Committee1, Sabin CA, Schwenk A, Johnson MA, Gazzard B, et al. (2010) Late diagnosis in the HAART era: proposed common definitions and associations with mortality. AIDS 24: 723-727.

23 http://www.who.int/hiv/pub/guidelines/hivstaging/en/

24 Mahajan AP, Sayles JN, Patel VA, Remien RH, Sawires SR, et al. (2008) Stigma in the HIV/AIDS epidemic: a review of the literature and recommendations for the way forward. AIDS 22: 67-79.

25 Genberg BL, Kawichai S, Chingono A, Sendah M, Chariyalertsak $S$, et al. (2008) Assessing HIV/AIDS stigma and discrimination in developing countries. AIDS Behav 12: 772-780.

26 Stangl Anne I, Brady I, Fritz K (2012) Measuring HIV stigma and discrimination: TECHNICAL BRIEF. Washington, Dc: International center for Research on Women.

27 Assefa Y, Jerene D, Lulseged S, Ooms G, Van Damme W (2009) Rapid scale-up of antiretroviral treatment in Ethiopia: successes and system-wide effects. PLoS Med 6: e1000056. 